\title{
Pertussis Surveillance in Veterans Affairs Medical Centers in Western United States - 2010-2014
}

\author{
Patricia Schirmer*1, Renee-Claude Mercier ${ }^{1,2}$, Cynthia A. Lucero-Obusan ${ }^{1}$, Gina Oda ${ }^{1}$ and \\ Mark Holodniy ${ }^{1,3}$
}

${ }^{1}$ Department of Veterans Affairs, Palo Alto, CA, USA; '2University of New Mexico, Albuquerque, NM, USA; ${ }^{3}$ Stanford University, Palo Alto, CA, USA

\section{Objective} US.

To perform pertussis surveillance in VA facilities in the Western

\section{Introduction}

Bordetella pertussis infection (whooping cough) has been on the rise and the most cases in the US since 1955 were reported in 2012 $(48,277 \text { or } 15.4 \text { per } 100,000)^{1}$. Pertussis is highly infectious and can cause serious illness in infants and children as well as adults, and in general is preventable by vaccination. Since 2005, it has been recommended that anyone 19-64 years old should have a onetime booster of the pertussis vaccine (Tdap). In 2010, that recommendation was broadened stating people 65 years old and older should also obtain a booster of Tdap. Given the increased number of pertussis cases in the Western US, and that approximately $20 \%$ of these cases occurred in patients $>20$ years of age, we performed pertussis surveillance in Veterans in care at VA medical facilities.

\section{Methods}

Pertussis cases from 1/1/2010-7/15/2014 were captured using VA's Healthcare Associated Infection and Influenza Surveillance System (HAIISS) with both QC Pathfinder (QCP) and VA ESSENCE applications for 14 states in the Western US. Patients were excluded if $<20$ years old. Laboratory tests for pertussis utilized in VA included culture, polymerase chain reaction (PCR), and pertussis-specific immunoglobulin tests. Using QCP, we defined laboratory confirmed pertussis cases as having a positive culture or PCR test from a respiratory site or a positive Bordetella IgM result, since the latter test was commonly used by providers. Inpatient and outpatient encounters coded with a pertussis ICD-9 code $(033.0,033.8$ or 033.9$)$ were identified using ESSENCE for the same time period. VA electronic medical records (EMR) were reviewed on all laboratory-confirmed or ICD-9 coded cases to confirm the diagnosis and determine TDaP vaccination status.

\section{Results}

A total of 383 potential pertussis cases were identified by HAIISS. EMR reviews determined that 108 did not have acute pertussis (98 received a pertussis ICD-9 code but there was no evidence of active infection either by laboratory results or documented symptoms; 3 had other Bordetella species in non-respiratory specimens; and 7 were under age 20). Of these excluded patients, 22 were identified as seeking pertussis prophylaxis for a recent exposure. Of the remaining 275 unique patients, 62 were identified with laboratory confirmed pertussis by QCP, 232 with pertussis ICD-9 codes in ESSENCE and 19 by both systems. The number of cases per year from 2010-2014 was $63,65,70,54$, and 23 , respectively. 49/275 (18\%) were female while 226/275 (82\%) were male. Median age of patients with acute pertussis was 55 years old (range 24-101). Figure 1 shows 5-year cumulative total of all cases by state and laboratory confirmed cases by VA facility. 28/275 (10\%) required hospitalization. The number of patients tested for pertussis was 119/275 (43\%). Tdap vaccination rates that were received or documented in VA's EMR (regardless of whether vaccine was given prior to encounter) was 148/275 (54\%). No patients died due to pertussis.

\section{Conclusions}

While pertussis is often thought of as a childhood illness, the VA is seeing a consistent number of cases in older adults each year. ICD-9 codes or laboratory positive cases alone are not reliable indicators of pertussis cases; surveillance was enhanced when both were used to identify cases. Mapping cases by residence zip code and by positive laboratory testing by facility showed that Utah has less ICD-9 coded visits, but a high number of laboratory confirmed cases while the opposite is seen in California. Vaccination and testing for pertussis in VA could be improved.

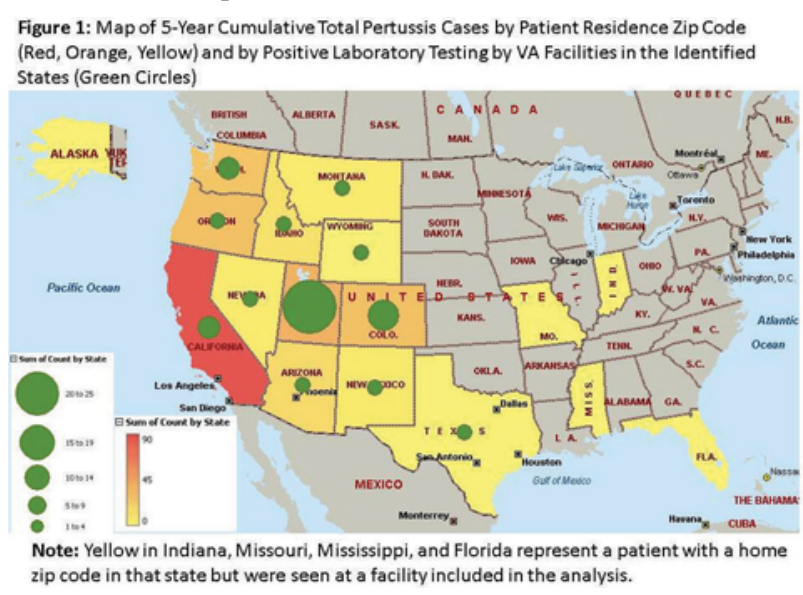

\section{Keywords}

Pertussis; Veterans; ESSENCE; Biosurveillance; ICD-9

\section{References}

1. CDC 2012 Pertussis Surveillance Report. August 23, 2013. Accessed September 3, 2014. Available at: http://www.cdc.gov/pertussis/ downloads/pertuss-surv-report-2012.pdf

\section{*Patricia Schirmer}

E-mail: Patricia.Schirmer@va.gov 